\title{
SPLUNC1 degradation by the cystic fibrosis mucosal environment drives airway surface liquid dehydration
}

\author{
Megan J. Webster ${ }^{1}$, Boris Reidel ${ }^{1}$, Chong D. Tan ${ }^{1}$, Arunava Ghosh ${ }^{1}$, \\ Neil E. Alexis ${ }^{2}$, Scott H. Donaldson ${ }^{1,3}$, Mehmet Kesimer ${ }^{1}$, Carla M.P. Ribeiro ${ }^{1,4}$ \\ and Robert Tarran ${ }^{1,4}$
}

Affiliations: ${ }^{1}$ Marsico Lung Institute, The University of North Carolina, Chapel Hill, NC, USA. ${ }^{2}$ Center for Asthma and Lung Biology, The University of North Carolina, Chapel Hill, NC, USA. ${ }^{3}$ Division of Pulmonary and Critical Care Medicine, The University of North Carolina, Chapel Hill, NC, USA. ${ }^{4}$ Dept of Cell Biology and Physiology, The University of North Carolina, Chapel Hill, NC, USA.

Correspondence: Robert Tarran, 7102 Marsico Hall, 125 Mason Farm Road, University of North Carolina, Chapel Hill, NC, 27599, USA. E-mail: robert_tarrandamed.unc.edu

\section{@ERSpublications}

The extracellular cystic fibrosis (CF) environment plays a key role in regulating transepithelial ion transport. CF secretions degrade SPLUNC1 and activate ENaC, causing airway dehydration. http://ow.ly/6WJA30lnDyC

Cite this article as: Webster MJ, Reidel B, Tan CD, et al. SPLUNC1 degradation by the cystic fibrosis mucosal environment drives airway surface liquid dehydration. Eur Respir J 2018; 52: 1800668 [https://doi. org/10.1183/13993003.00668-2018].

ABSTRACT The multi-organ disease cystic fibrosis (CF) is caused by mutations in the cystic fibrosis transmembrane regulator gene (CFTR) that lead to diminished transepithelial anion transport. CF lungs are characterised by airway surface liquid (ASL) dehydration, chronic infection/inflammation and neutrophilia. Dysfunctional CFTR may upregulate the epithelial $\mathrm{Na}^{+}$channel $(\mathrm{ENaC})$, further exacerbating dehydration. We previously demonstrated that short palate lung and nasal epithelial clone 1 (SPLUNC1) negatively regulates $\mathrm{ENaC}$ in normal airway epithelia.

Here, we used pulmonary tissue samples, sputum and human bronchial epithelial cells (HBECs) to determine whether SPLUNC1 could regulate $\mathrm{ENaC}$ in a CF-like environment.

We found reduced endogenous SPLUNC1 in CF secretions, and rapid degradation of recombinant SPLUNC1 (rSPLUNC1) by CF secretions. Normal sputum, containing SPLUNC1 and SPLUNC1-derived peptides, inhibited ENaC in both normal and CF HBECs. Conversely, CF sputum activated ENaC, and rSPLUNC1 could not reverse this phenomenon. Additionally, we observed upregulation of ENaC protein levels in human CF bronchi. Unlike SPLUNC1, the novel SPLUNC1-derived peptide SPX-101 resisted protease degradation, bound apically to HBECs, inhibited $\mathrm{ENaC}$ and prevented ASL dehydration following extended pre-incubation with CF sputum.

Our data indicate that CF mucosal secretions drive ASL hyperabsorption and that protease-resistant peptides, e.g. SPX-101, can reverse this effect to rehydrate CF ASL. 


\section{Introduction}

Cystic fibrosis (CF) is a genetic, multi-organ disease caused by mutations in the cystic fibrosis transmembrane conductance regulator gene (CFTR) that result in defective transepithelial anion transport [1]. In the airways, the epithelial $\mathrm{Na}^{+}$channel $(\mathrm{ENaC})$ is the rate-limiting step that governs transepithelial $\mathrm{Na}^{+}$absorption [2]. The absence of CFTR leads to increased $\mathrm{ENaC}$ activity and $\mathrm{Na}^{+}$hyperabsorption, which in combination with anion hyposecretion causes ASL dehydration, thus reducing mucociliary clearance [3-5]. Together, these changes are predicted to cause the accumulation of dehydrated intraluminal mucus plugs that serve as a nidus for subsequent bacterial infections. A failure to resolve these infections causes chronic inflammation/neutrophilia and elevated lung luminal protease levels. These proteases, including neutrophil elastase (NE) and cathepsins B and S, then cause bronchiectasis and ultimately destroy the lung [6,7]. A correlation between NE activity and CF lung disease severity has recently been reported [8].

Short palate lung and nasal epithelial clone 1 (SPLUNC1) is a multifunctional $25 \mathrm{kDa}$ innate defence protein that is secreted into the airway surface liquid (ASL) [9-12]. SPLUNC1 is an allosteric regulator that binds extracellularly, causing a conformational change to ENaC. This binding event is followed by NEDD4.2-dependent ubiquitination of $\alpha$-ENaC and subsequent internalisation of the channel, which serves to reduce $\mathrm{Na}^{+}$and ASL absorption [11-13]. Resolution of SPLUNC1's crystal structure has allowed its functions to be assigned to distinct regions. For example, the N-terminal "S18" region inhibits the $\mathrm{ENaC}$ to modulate ASL volume $[11,12]$.

Interactions between CFTR and $\mathrm{ENaC}$ have been reported in heterologous expression systems [4, 14, 15]. However, the nature of these interactions and the underlying mechanism of $\mathrm{Na}^{+}$hyperabsorption are unknown. As such, the role of $\mathrm{ENaC}$ in $\mathrm{CF}$ lung pathophysiology remains controversial [16, 17]. SPLUNC1 inhibits ENaC in normal ASL. However, due to a series of $\mathrm{pH}$-sensitive salt bridges, SPLUNC1 fails to function in moderately acidic CF ASL ( $\sim \mathrm{pH} 6.5)$ [11]. Thus, under thin film conditions where native ASL has accumulated, SPLUNC1 can spontaneously inhibit ENaC in normal but not CF epithelia. Because they lack the $\mathrm{pH}$-sensitive salt bridges, peptides of SPLUNC1's ENaC-inhibitory domain are $\mathrm{pH}$ independent and inhibit ENaC in CF-affected epithelia [12]. For example, a SPLUNC1 peptidomimetic, SPX-101, is currently undergoing clinical trials for the treatment of CF lung disease [18]. We have previously focused on the initiating events in CF $[5,11]$. However, given the high levels of neutrophilia and protease activity within CF airways, we postulated that endogenous SPLUNC1 was susceptible to non-pH-related degradation in CF lungs, which, if it occurred within the S18 region, would prevent ENaC inhibition. Thus, using patient-derived samples, we tested the hypotheses that 1) SPLUNC1 is degraded in $\mathrm{CF}$ airways, leading to increased ENaC activity; and 2) SPX-101 can overcome this deficiency to induce $\mathrm{ENaC}$ inhibition and rehydrate CF ASL.

\section{Methods}

A detailed description of the methods used is provided in the supplementary material.

\section{Normal/CF sputum and supernatant of mucopurulent material}

Induced and spontaneous sputum samples were obtained as described [9]. Supernatant of mucopurulent material (SMM) was harvested from the airways of excised human CF lungs as described [19]. Donor demographics are shown in supplementary tables S1, S2 and S3.

\section{Proteins and peptides}

SPLUNC1 was expressed and purified as described previously [11]. SPX-101 (aaLPIPLDQTaa) was prepared by solid-state fluorenylmethyloxycarbonyl (Fmoc) synthesis as described previously [18].

\section{Western blot}

Endogenous NE and SPLUNC1 protein levels were determined using neat sputum samples from 18 donors. For degradation experiments, PBS, NE, normal or CF sputa (pooled from $\mathrm{n}=6$ donors per group) and SMM (pooled from $\mathrm{n}=3$ donors) were co-incubated with $10 \mu \mathrm{M}$ rSPLUNC1 at $37^{\circ} \mathrm{C}$. Where applicable, inhibitors were pre-incubated with sputa for $1 \mathrm{~h}$ prior to rSPLUNC1 incubation.

\section{NE activity assay}

NE activity levels were determined using the NE-sensitive peptide Suc-Ala-Ala-Ala-7-amino-4methylcoumarin (MCA) as described in the supplementary material.

\section{ENaC and $\mathrm{Na}^{+} / \mathrm{K}^{+}$-ATPase expression in human bronchi}

Human lungs were obtained as described above; donor demographics are shown in supplementary table S3. Bronchi were dissected from the underlying tissue; CF bronchi were selected from relatively disease-free 
regions. Tissues were rinsed using a lactated Ringer's solution and proteins extracted using lysis buffer containing NP40 [20].

\section{Determination of rSPLUNC1 and SPX-101 cleavage by mass spectrometry}

$10 \mu \mathrm{M}$ of rSPLUNC1 and SPX-101 were incubated with pooled normal or CF sputa ( $\mathrm{n}=6$ donors per group). Samples were snap-frozen at $-80^{\circ} \mathrm{C}$ and prepared by filter-aided sample preparation for proteomics [21]. Solubilised peptide material was injected for label-free quantitative proteomic analysis utilising a Q Exactive (Thermo Scientific, Waltham, MA, USA) mass spectrometer coupled to an UltiMate 3000 (Thermo Scientific) nanoHPLC system, and data acquisition was performed as described [22].

\section{HBEC culture}

Cells were harvested from human lungs deemed unsuitable for transplantation (non-CF donors) or post-transplantation (CF donors) as per University of North Carolina (UNC) protocol \#03-1396. They were cultured as described on permeable supports and maintained at the air-liquid interface in a modified bronchial epithelial growth medium [20]. Cells were studied 21-28 days after seeding.

\section{ASL height measurements}

$30 \mu \mathrm{M}$ of rSPLUNC1 or SPX-101 were co-incubated with PBS or pooled normal/CF sputa ( $\mathrm{n}=6$ donors per group) overnight at $37^{\circ} \mathrm{C}$ with or without sivelestat before being added to HBEC mucosal surfaces. ASL heights were measured as described previously [11].

\section{Binding of SPLUNC1 and SPX-101 to HBECs}

Cells were exposed apically to either amine-reactive Dylight-633 rSPLUNC1 or 5-TAMRA-SPX-101 preincubated with PBS, normal or CF pooled sputa. Cells were counterstained using calcein-acetoxymethyl (AM) and imaged using an SP8 confocal microscope (Leica Microsystems, Wetzlar, Germany).

\section{Transepithelial potential difference}

A single-barrelled transepithelial potential difference $\left(\mathrm{V}_{\mathrm{t}}\right)$-sensing microelectrode was positioned in the ASL by a micromanipulator and used in conjunction with a macroelectrode in the serosal solution to measure Vt using a voltmeter (World Precision Instruments, Sarasota, FL, USA) as described [11, 23].

\section{Statistics}

Normally distributed data were analysed using ANOVA followed by Tukey's test or a t-test. Non-parametric equivalents (Mann-Whitney U test, Kruskal-Wallis test with Dunn's multiple comparisons test) were used when data were not normally distributed. For SPLUNC1 degradation over time, curves were fit with single exponentials and analysed using the extra sum of squares $\mathrm{F}$ test. Data analysis was performed using GraphPad Prism 7.0 (GraphPad Software, La Jolla, CA, USA).
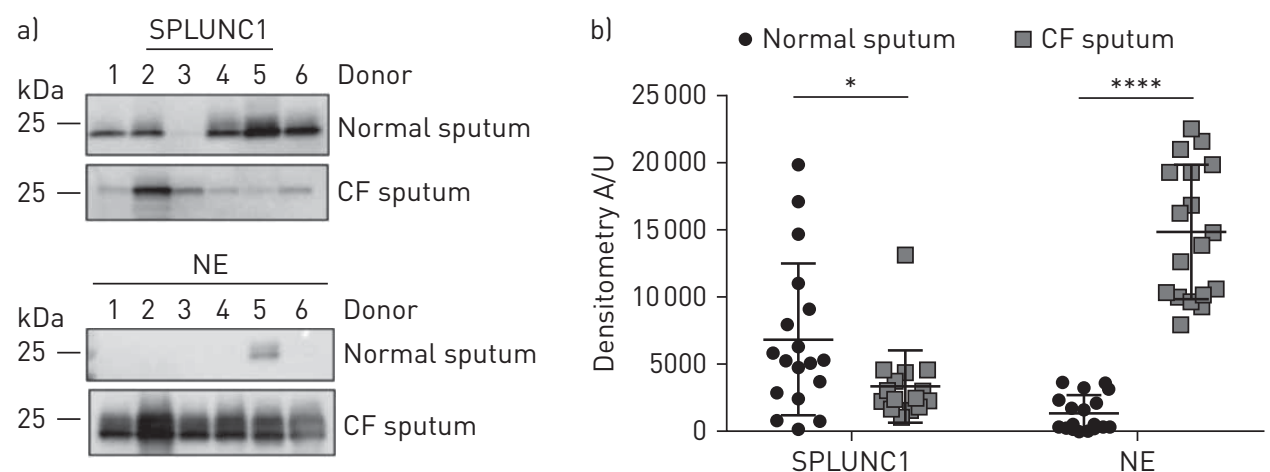

FIGURE 1 SPLUNC1 protein levels are significantly reduced in cystic fibrosis (CF) sputum. a) Representative Western blots showing endogenous expression of SPLUNC1 and neutrophil elastase (NE) in normal and CF sputum samples from $n=6$ donors per group. Membranes were probed for NE prior to stripping and re-probing for SPLUNC1. b) Densitometrical analysis of SPLUNC1 and NE protein abundance in normal and CF sputum from $n=18$ donors per group. Data are presented as mean \pm SD. Data were analysed by $t$-test with Welch's correction. *: $p<0.05$, SPLUNC1 normal versus CF sputum; ${ }^{* * * *}$ : $p<0.0001$, NE normal versus CF sputum. 


\section{Results}

\section{SPLUNC1 protein levels are decreased in CF sputum}

Consistent with previous studies, we detected SPLUNC1 levels by Western blot analysis in normal sputum samples $[9,24]$. We detected a significant reduction in endogenous SPLUNC1 protein levels in CF sputum (figure 1). Because NE can degrade SPLUNC1 [24], we next determined NE protein levels. In agreement with previous studies [25], we found increased NE levels in CF sputum (figure 1). We next co-incubated rSPLUNC1 with pooled sputa from normal and CF donors. Full-length rSPLUNC1 was exponentially degraded by $C F$ sputum $\left(\mathrm{t}_{1 / 2}=26 \mathrm{~min}\right)$, but remained stable following incubation with normal sputum (figure 2a, b). Because CF lung disease increases the ASL protein content, we co-incubated rSPLUNC1 with volume-adjusted normal and CF sputa to test for rSPLUNC1 degradation independently of protein concentrations. rSPLUNC1 levels were significantly reduced in the presence of CF sputum only (figure $2 \mathrm{c}, \mathrm{d}$ ). To confirm that the degradation of SPLUNC1 was protein mediated, sputum samples were incubated with normal or $\mathrm{CF}$ sputum at $4^{\circ} \mathrm{C}$ or in the presence of heat-denatured normal and $\mathrm{CF}$ sputum $\left(95^{\circ} \mathrm{C} /\right.$ $\beta$-mercaptoethanol). Both of these manoeuvres attenuated rSPLUNC1 degradation in CF sputum (supplementary figure S1a, b). Given that collection methods differed for normal and CF sputa (i.e. induced versus spontaneous collection, respectively), we confirmed that rSPLUNC1 stability was unaffected by the collection method and we found no difference in SPLUNC1 stability in spontaneous versus induced sputum (supplementary figure S2).

\section{NE in CF sputum is partially responsible for SPLUNC1 degradation}

We co-incubated rSPLUNC1 and CF sputum with inhibitors of several proteases, their respective inhibitors and an EDTA-free protease inhibitor cocktail (PIC), used to inhibit multiple proteases [19]. Only sivelestat and 10× PIC significantly attenuated rSPLUNC1 degradation (figure 3a, b). Because NE was elevated in CF sputum, and SPLUNC1's degradation was sivelestat sensitive, we co-incubated rSPLUNC1 with NE, which degraded rSPLUNC1 in a concentration-dependent manner (figure $3 \mathrm{c}, \mathrm{d}$ ). Owing to the potential importance of these findings, we exposed rSPLUNC1 to normal or CF sputa in the presence of protease inhibitors for extended times. Surprisingly, sivelestat was unable to prevent rSPLUNC1 degradation after extended incubation periods (figure $4 \mathrm{a}, \mathrm{b}$ ).

a)
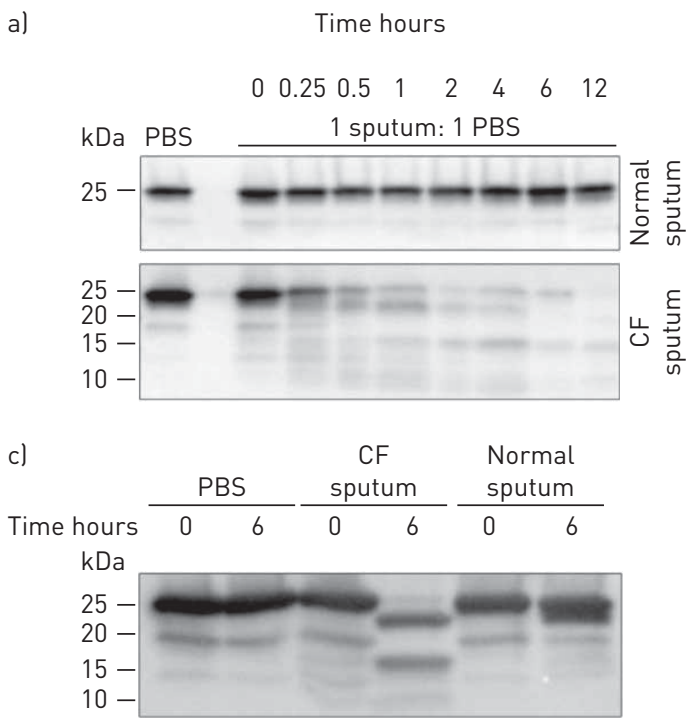
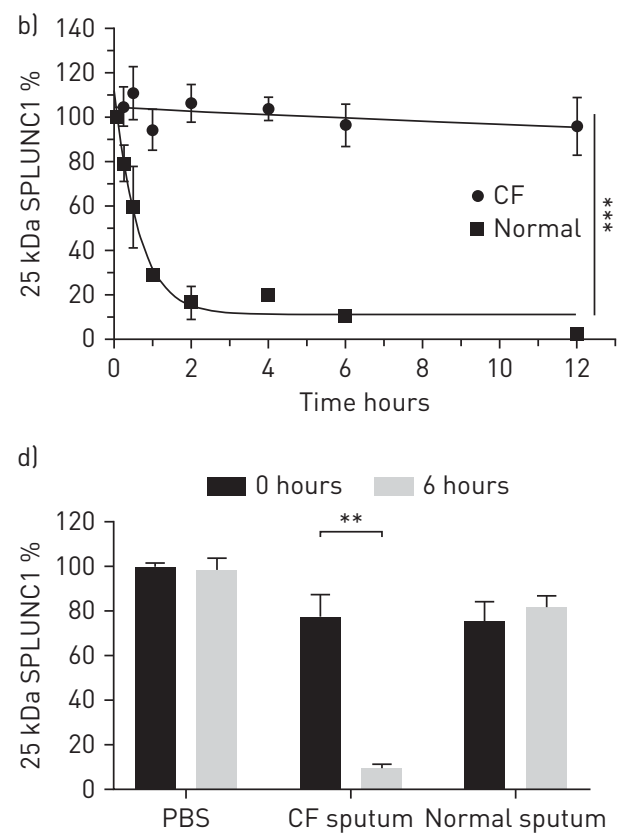

FIGURE 2 rSPLUNC1 is rapidly degraded in cystic fibrosis (CF) sputum. a) Representative Western blots showing SPLUNC1 abundance after incubation with CF and normal sputum (pooled from $\mathrm{n}=6$ donors) at $37^{\circ} \mathrm{C}$. b) Densitometrical analyses showing rapid degradation of rSPLUNC1. Data are presented as the mean \pm SD of $n=3-4$ individual experiments. The difference between the rate constant ( $k$ ) for PBS versus supernatant of mucopurulent material (SMM) rSPLUNC1 degradation was analysed by extra sum of squares F-test; ***: $p<0.001$. CF sputum: $t_{1 / 2}=\sim 26$ min, $k=1.57$; normal sputum: $t_{1 / 2}=\sim 4 h, k=0.02$. c) Representative Western blot showing significant degradation of $10 \mu \mathrm{M}$ rSPLUNC1 by CF sputum following a $6 \mathrm{~h}$ incubation at $37^{\circ} \mathrm{C}$, with $15 \mu \mathrm{g}$ of total protein and d) associated densitometry; data are presented as mean \pm SEM for $n=3-5$ individual experiments. Data were analysed by Mann-Whitney U test; **: $p<0.01$. 

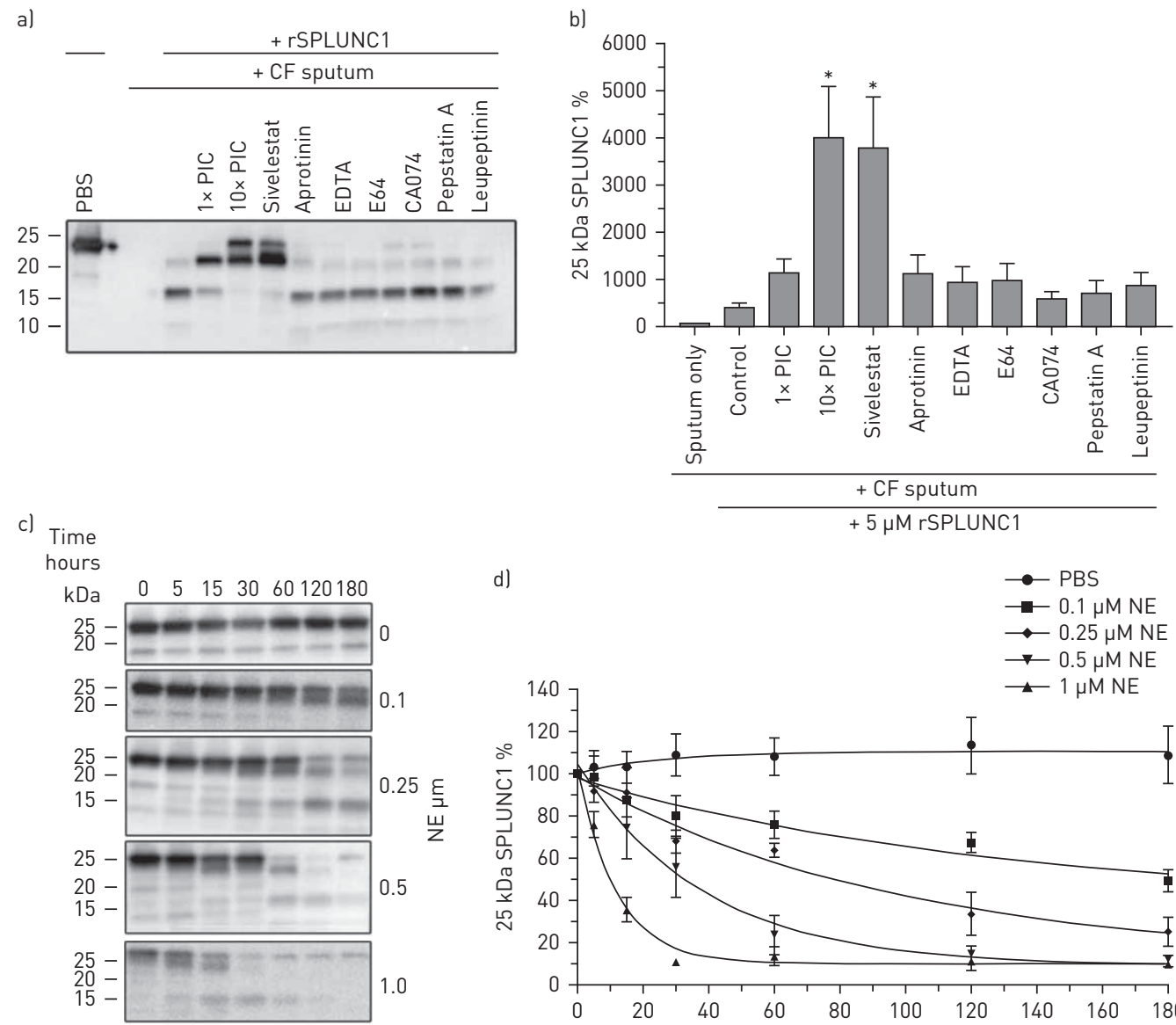

FIGURE 3 Sivelestat partially attenuates rSPLUNC1 degradation. a) Representative Western blot showing $25 \mathrm{kDa}$ rSPLUNC1 abundance following co-incubation with $1 \times / 10 \times$ protease inhibitor cocktail (PIC) or $10 \mu \mathrm{M}$ protease inhibitors and cystic fibrosis (CF) sputum (pooled from $n=6$ donors) for $6 \mathrm{~h}$ and $\mathrm{b}$ ) associated densitometry from $\mathrm{n}=8$ individual experiments; data are presented as mean $\pm \mathrm{SEM}, \% 25 \mathrm{kDa}$ SPLUNC1 expression compared with CF sputum alone. Data show significant inhibition of rSPLUNC1 degradation by $10 \mu \mathrm{M}$ sivelestat and 10x PIC only. Data were analysed by Kruskal-Wallis test; *: $p<0.05$. c) Representative Western blots following exposure of rSPLUNC1 to neutrophil elastase (NE) over time and d) associated densitometry from $\mathrm{n}=4-6$ individual experiments. Data are presented as mean \pm SEM. Data were analysed by extra sum of squares F-test; $p<0.0001$, rate constant ( $k$ ) different between each NE data set.

To confirm the translation of high NE protein levels into elevated NE activity, we added a NE-sensitive substrate to sputum \pm sivelestat, and measured the formation of the fluorescent product AMC, which was significantly elevated after exposure to CF but not normal sputum (figure 4c). Sivelestat was unable to reduce AMC formation following $12 \mathrm{~h}$ of exposure to $\mathrm{CF}$ sputum (figure $4 \mathrm{~d}$ ) and failed to recover SPLUNC1's ability to regulate ASL height (supplementary figure S3a, b). Additionally, we tested whether cathepsins B, K, S and L were active in CF sputum. Using protease-specific fluorogenic substrates, we observed significantly greater cathepsin $\mathrm{B}$, cathepsin $\mathrm{K}$ and cathepsin $\mathrm{S} / \mathrm{L}$ activity in CF relative to normal sputum (supplementary figure $\mathrm{S} 4 \mathrm{a}-\mathrm{c}$ ). We also examined MMP-2/9 activity, which was significantly elevated in CF sputum (supplementary figure S4d). Because the inhibitors of these enzymes failed to attenuate rSPLUNC1 degradation, we conclude that whilst a number of proteases are functionally active in CF sputum, NE is predominantly responsible for rSPLUNC1 degradation.

\section{Upregulation of $\alpha-, \beta$ - and $\gamma-E N a C$ and $\mathrm{Na}^{+} / \mathrm{K}^{+}$ATPase $\alpha 1$ protein expression in $\mathrm{CF}$ bronchi indicates increased $\mathrm{ENaC}$ activity}

SPLUNC1 was undetectable in SMM collected from excised CF lungs (figure 5a). Similarly, co-incubation of SMM and rSPLUNC1 resulted in a rapid diminution of rSPLUNC1 (figure 5b, c). Because SPLUNC1 affects $\mathrm{ENaC}$ proteostasis [13], we measured $\mathrm{ENaC}$ levels. Bronchi were collected from non-CF and relatively disease-free areas of CF lungs and we measured protein levels by Western blot [11]. We observed a significant increase in all $\mathrm{ENaC}$ subunits in $\mathrm{CF}$ bronchi (figure $5 \mathrm{~d}, \mathrm{e}$ ). $\alpha$ - and $\gamma$-ENaC must be proteolytically cleaved before $\mathrm{ENaC}$ can conduct $\mathrm{Na}^{+}$[26]. Interestingly, we detected significant increases 
a)
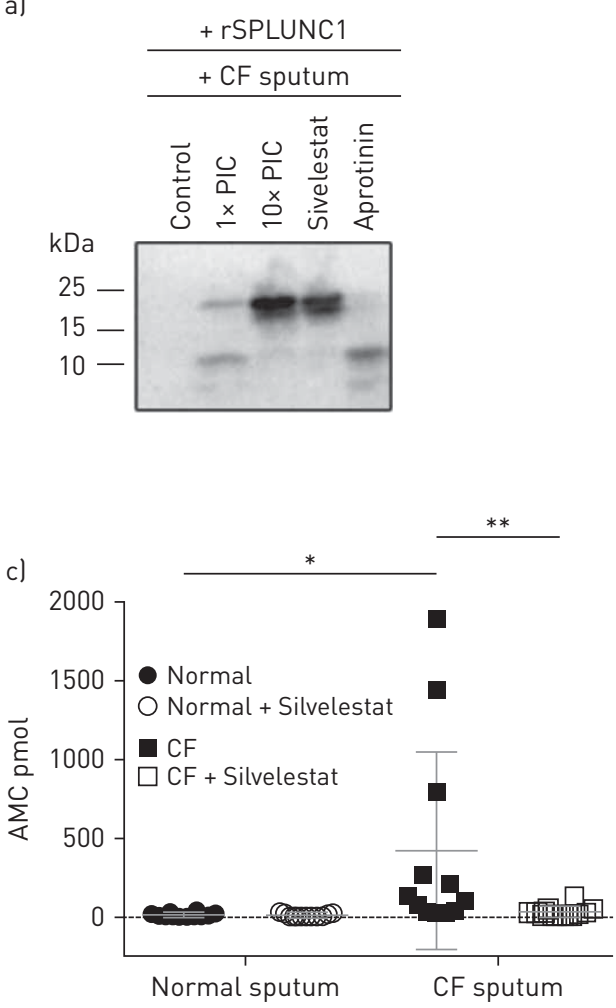

b)

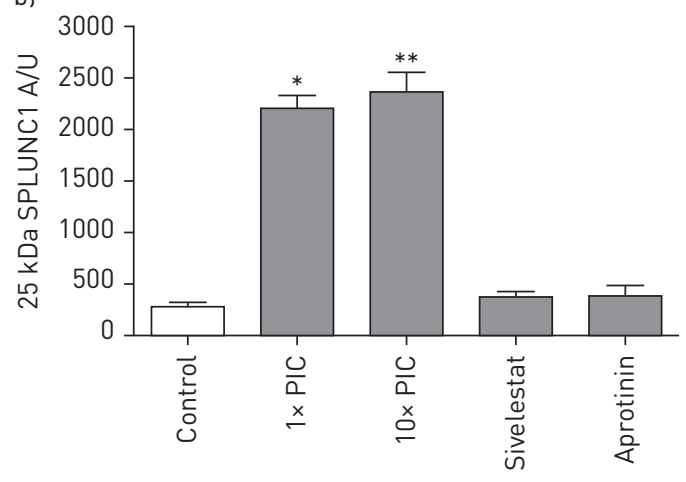

+ CF sputum and rSPLUNC1

d)

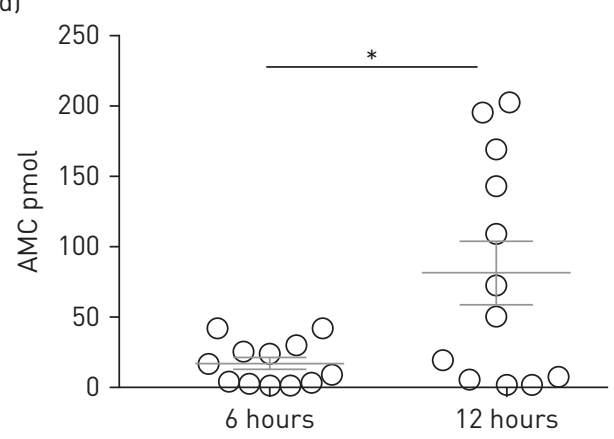

FIGURE 4 Sivelestat fails to prevent rSPLUNC1 degradation by cystic fibrosis (CF) sputum over extended periods. a) Representative Western blot showing $25 \mathrm{kDa}$ rSPLUNC1 abundance following co-incubation of $1 \times / 10 \times$ protease inhibitor cocktail (PIC), $10 \mu \mathrm{M}$ sivelestat or $10 \mu \mathrm{M}$ aprotinin and CF sputum (pooled from $\mathrm{n}=6$ donors) and b) associated densitometry from $\mathrm{n}=3-4$ individual experiments; data are presented as mean \pm SEM. $\% 25 \mathrm{kDa}$ SPLUNC1 was compared with CF sputum only by Kruskal-Wallis test; *: $p<0.05$; **: $p<0.01$. c) 7-amino-4-methylcoumarin (AMC) formation following cleavage of $100 \mu \mathrm{M}$ fluorogenic substrate Suc-Ala-Ala-Ala-MCA after $6 \mathrm{~h}$ incubation with normal and $\mathrm{CF}$ sputum at $37^{\circ} \mathrm{C} \pm 10 \mu \mathrm{M}$ sivelestat. Data are presented as mean \pm SD from $n=12$ normal and $n=12$ CF donors. Kruskal-Wallis test with Dunn's multiple comparison for normal versus CF, *: $p<0.05$; for CF versus CF+sivelestat, ${ }^{* *}: p<0.01$. d) Change in AMC formation after cleavage of $100 \mu \mathrm{M}$ fluorogenic substrate Suc-Ala-Ala-Ala-MCA by CF sputum after 6 or $12 \mathrm{~h}$ $+10 \mu \mathrm{M}$ sivelestat. Data are presented as mean \pm SD from $n=12$ normal and $n=12$ CF donors. Data were analysed by Mann-Whitney U test; *: p<0.05.

in cleaved $\mathrm{ENaC}$ subunits in $\mathrm{CF}$ bronchi, suggesting that $\mathrm{ENaC}$ activity is upregulated (figure $5 \mathrm{~d}, \mathrm{e}$ ). Consistent with increased $\mathrm{ENaC}$ levels, the $\mathrm{Na}^{+} / \mathrm{K}^{+}$ATPase $\alpha 1$ subunit was also increased in CF bronchi (figure $5 \mathrm{~d}, \mathrm{e}$ ).

\section{CF sputum cleaves the ENaC-inhibitory domain of SPLUNC1}

$\mathrm{NE}$ predominantly cleaves valine and alanine residues. Indeed, co-incubation of rSPLUNC1 with CF but not normal sputum generated elastase-specific peptides (figure 6a). As a control, we performed a tryptic digest, and detected peptides regardless of genotype (figure $6 \mathrm{~b}$ ). Coverage analysis revealed valine-specific cleavage of SPLUNC1's S18 region (GGLPVPLDQTLPLNVPA) and the formation of PLDQTLPLNV, which is likely unable to regulate $\mathrm{ENaC}$ (figure 6c). We recently developed a novel, NE-resistant, SPLUNC1 peptidomimetic called SPX-101 [18]. The ENaC interacting region of the SPX-101 peptide remained intact following exposure to CF sputum (figure 6c). To determine if rSPLUNC1 and SPX-101 remained active in the presence of CF sputum, we co-incubated PBS or sputum with rSPLUNC1 or SPX-101 overnight and added this apically to HBECs from normal donors. rSPLUNC1 and SPX-101 increased ASL height in the presence of PBS and normal sputum (figure 6d, e). Consistent with mass spectrometry data, only SPX-101 was capable of regulating ASL height in the presence of CF sputum (figure 6d, e).

\section{CF sputum prevents SPLUNC1 binding and elevates ENaC activity}

Because ENaC is apically expressed, SPLUNC1 must also bind apically as part of the inhibition process [13]. We therefore co-incubated fluorescently labelled rSPLUNC1 and SPX-101 with PBS or sputum, 

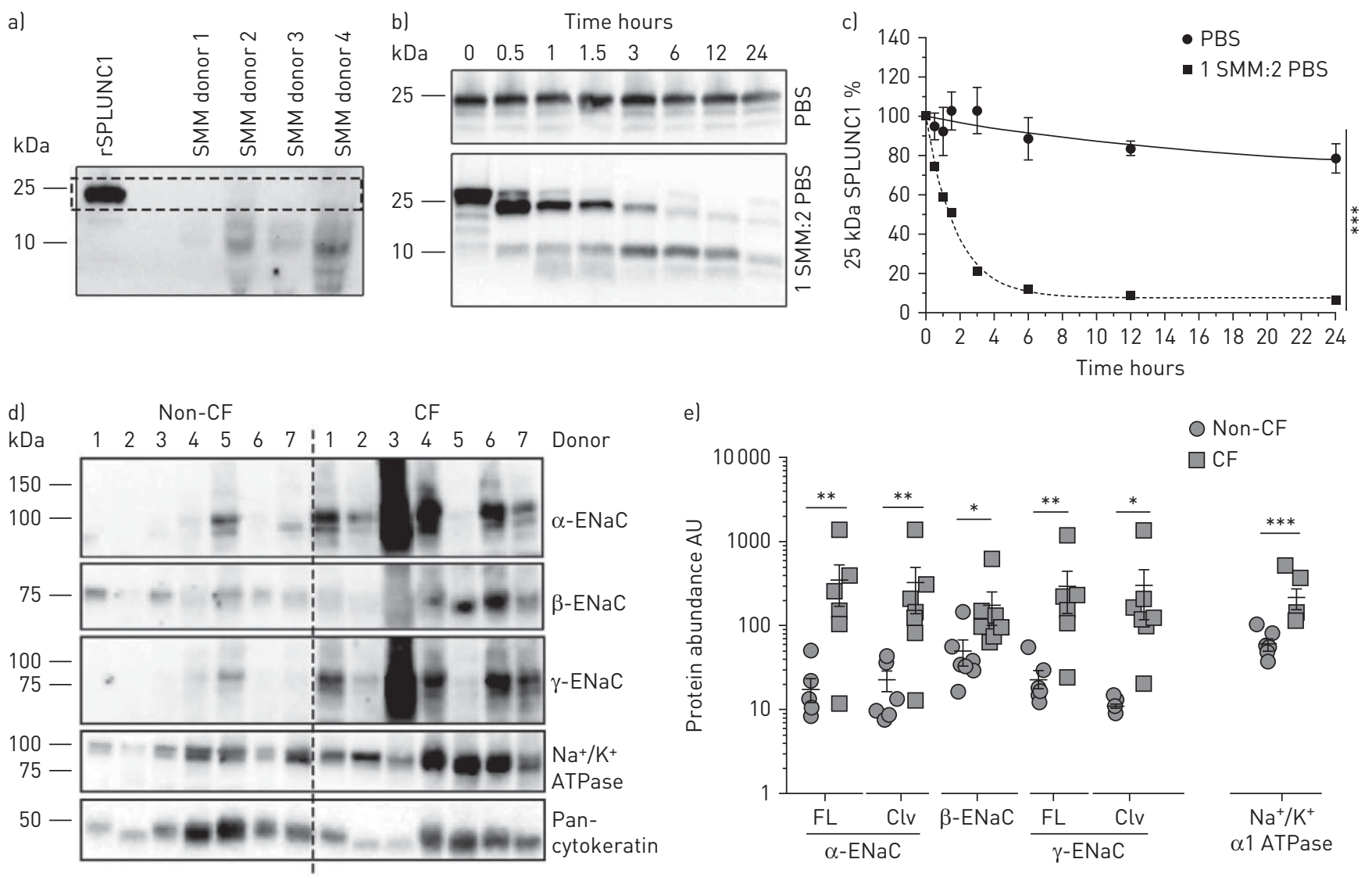

FIGURE 5 SPLUNC1 is degraded and $\alpha-, \beta$ - and $\gamma$-epithelial $\mathrm{Na}^{+}$channel (ENaC) subunits are upregulated in cystic fibrosis (CF) bronchi. a) Western blot showing endogenous SPLUNC1 levels in supernatant of mucopurulent material (SMM) extracted from $\mathrm{n}=4$ individual CF donor lungs obtained post-transplant. b) Representative Western blots showing $10 \mu \mathrm{M}$ rSPLUNC1 levels over time in PBS or SMM diluted 1:2 in PBS (SMM pooled $n=3-4$ donors). c) Associated densitometry of $25 \mathrm{kDa}$ SPLUNC1 from $\mathrm{n}=3$ independent experiments; data are presented as mean \pm SD. The rate constant $(k)$ for PBS versus SMM rSPLUNC1 degradation was analysed by extra sum of squares $F$-test; ${ }^{* * *}: p<0.001$. PBS: $t_{1 / 2}=14.6 \mathrm{~h}$, $\mathrm{k}=0.05$; SMM: $\mathrm{t}_{1 / 2}=1.2 \mathrm{~h}, \mathrm{k}=0.60$. d) Western blot showing abundance of full-length (FL) and cleaved (Clv) $\alpha-, \beta-$ and $\gamma-\mathrm{ENaC}$, as well as the $\mathrm{Na}^{+} / \mathrm{K}^{+}$ ATPase and pan-cytokeratin in bronchi dissected from $n=7$ non-CF and $n=7$ CF lungs post mortem or post-transplant. e) Associated densitometry; data are presented as mean \pm SD, $\log _{10}$ transformed. Protein abundance was normalised to pan-cytokeratin expression for each individual donor. Normal versus CF expression was analysed by Mann-Whitney U test; ${ }^{*}: p<0.05 ;{ }^{* *}: p<0.01 ;{ }^{* * *}: p<0.001$.

before apical addition to HBECs. We observed apical binding of Dylight633-rSPLUNC1 and 5-TAMRA-SPX-101 in both PBS- and normal sputa-treated HBECs (figure 7a). After exposure to CF sputum, SPX-101 but not SPLUNC1 was able to bind apically (figure 7a). Furthermore, SPX-101 but not SPLUNC1 remained functionally active and internalised $\alpha$-ENaC-GFP following exposure to NE (supplementary figure S5).

We next determined whether SPLUNC1 degradation by CF secretions contributed to ENaC hyperactivity. We have previously reported increases in ENaC activity in primary CF HBECs [23]. However, passaged HBECs do not display this phenomenon. To start with similar transepithelial voltages $(\mathrm{Vt}$ ), we exposed passaged normal and CF HBECs to normal or CF sputum, or PBS (control), and measured the resultant amiloride-sensitive $\mathrm{Vt}$ as a marker of ENaC activity. Baseline amiloride-sensitive Vts were not significantly different between normal and CF HBECs (figure 7b, c). However, adding normal sputum reduced $\mathrm{Vt}$ whilst CF sputum significantly elevated $\mathrm{Vt}$, independently of HBEC genotype. Addition of rSPLUNC1 in PBS significantly reduced $\mathrm{Vt}$ in normal but not CF HBECs, consistent with our previous findings that SPLUNC1 cannot function in CF HBECs [11]. rSPLUNC1 had no further effect on the already reduced Vt in HBECs exposed to normal sputum, suggesting that endogenous S18-like peptides were present in sufficient quantities to inhibit ENaC as described [12]. Normal but not CF sputum was able to significantly decrease the CF Vt (figure 7c), suggesting that spontaneously produced bioactive peptides are present in normal but not CF sputum. To further examine this effect, we passed normal sputum through a size-exclusion column to remove 

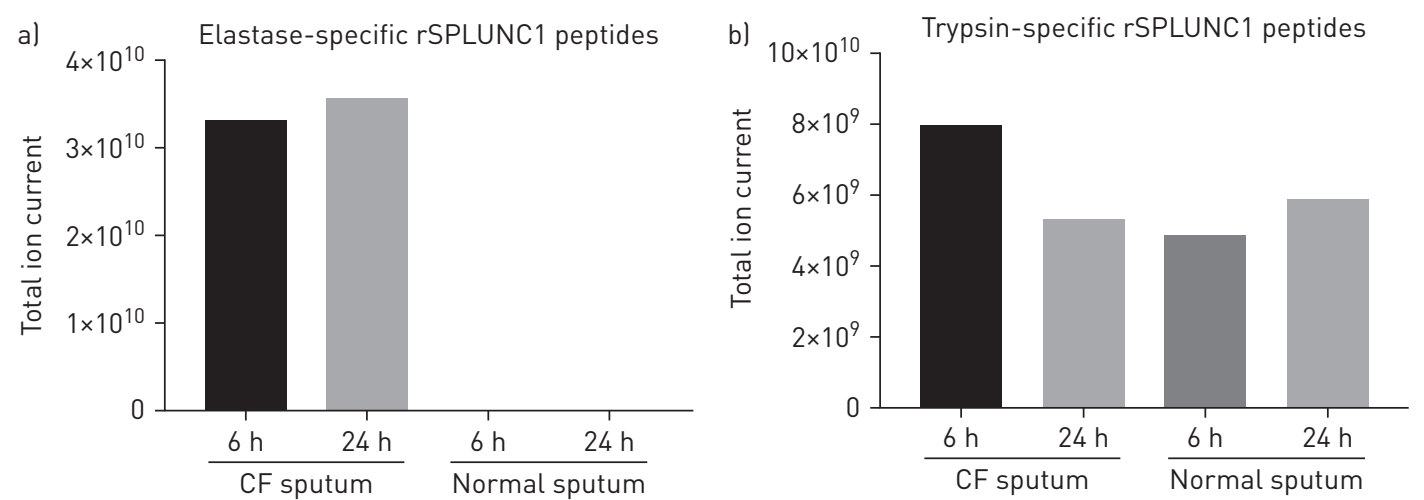

c)

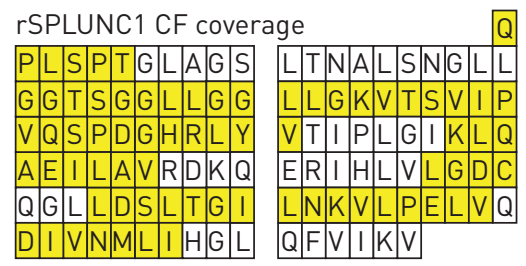

SPX-101 CF coverage
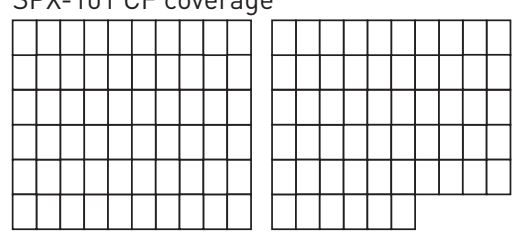

[FG $\mid$ G

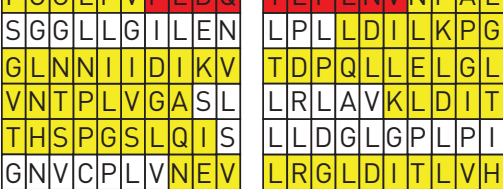

GNNVICP L V V N E V

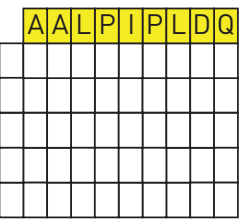

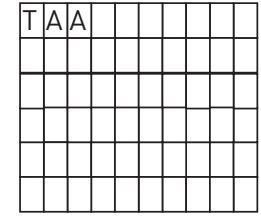

e)
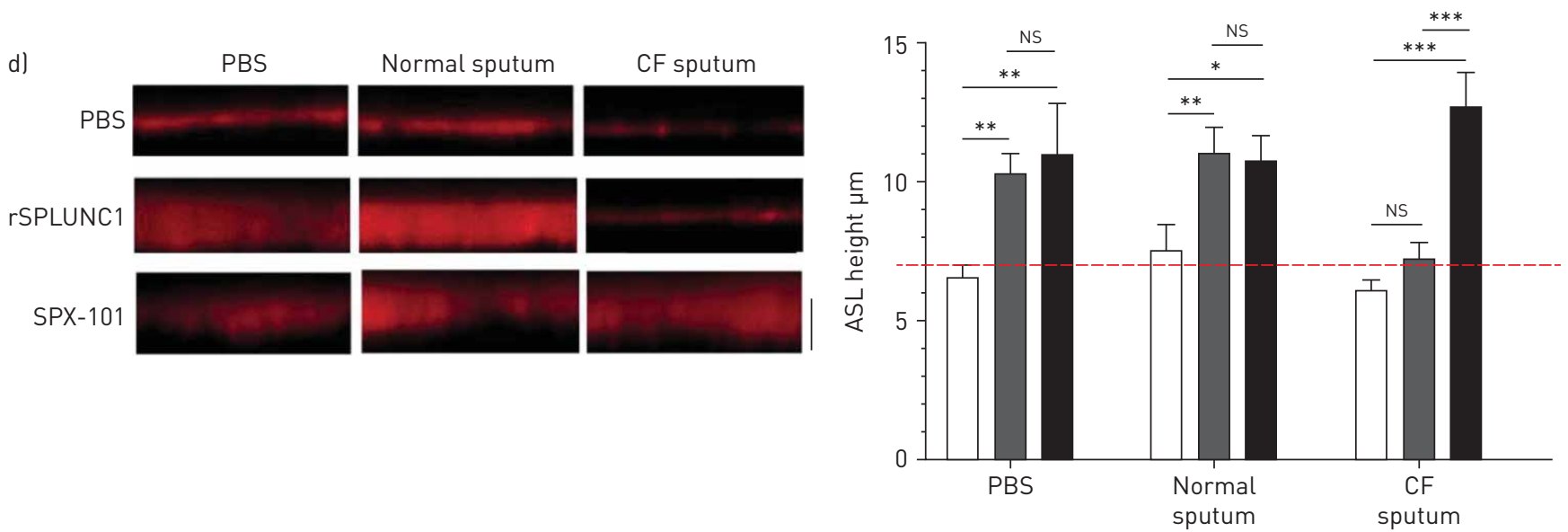

FIGURE 6 Cystic fibrosis (CF) sputum cleaves SPLUNC1's epithelial $\mathrm{Na}^{+}$channel (ENaC) inhibitory region. a, b) Total ion chromatogram intensities for elastase-specific (a) and trypsin-specific (b) SPLUNC1 peptides following incubation with CF and normal sputum at $37^{\circ} \mathrm{C}$. C) SPLUNC1 coverage after proteomic analysis of elastase-specific peptides. The peptide identified as PLDQTLPLNV indicating disruption of SPLUNC1's S18 region is highlighted in red. d) Representative airway surface liquid (ASL) heights following a $2 \mathrm{~h}$ incubation of human bronchial epithelial cells (HBECs) with PBS, normal sputum or CF sputum (pooled $n=6$ donors) $\pm 30 \mu M$ rSPLUNC1 or $30 \mu M$ SPX-101. e) ASL height measurements from $n=12-15$ transwells cultured from $n=4-5$ individual donors, presented as mean \pm SEM. Data were analysed by two-way ANOVA analysis with Tukey's post hoc analyses; ${ }^{*}: p<0.05 ;{ }^{* *}: p<0.01{ }^{* * *}$ : $p<0.001$; Ns: nonsignificant.

peptides and proteins $\geqslant 10 \mathrm{kDa}$. We then added this filtrate to normal HBECs and measured ASL height $6 \mathrm{~h}$ later. This filtrate significantly increased ASL height and adding $30 \mu \mathrm{M}$ rSPLUNC1 to the filtrate had no further effect (supplementary figure S6). Consistent with our previous observation that full-length SPLUNC1 is inactive in CF airways, rSPLUNC1 failed to reduce amiloride-sensitive Vt in the presence of CF sputum. SPX-101 was able to significantly inhibit the amiloride-sensitive Vt in both normal and CF HBECs after exposure to either PBS or CF sputum (figure $7 b, c$ ). 

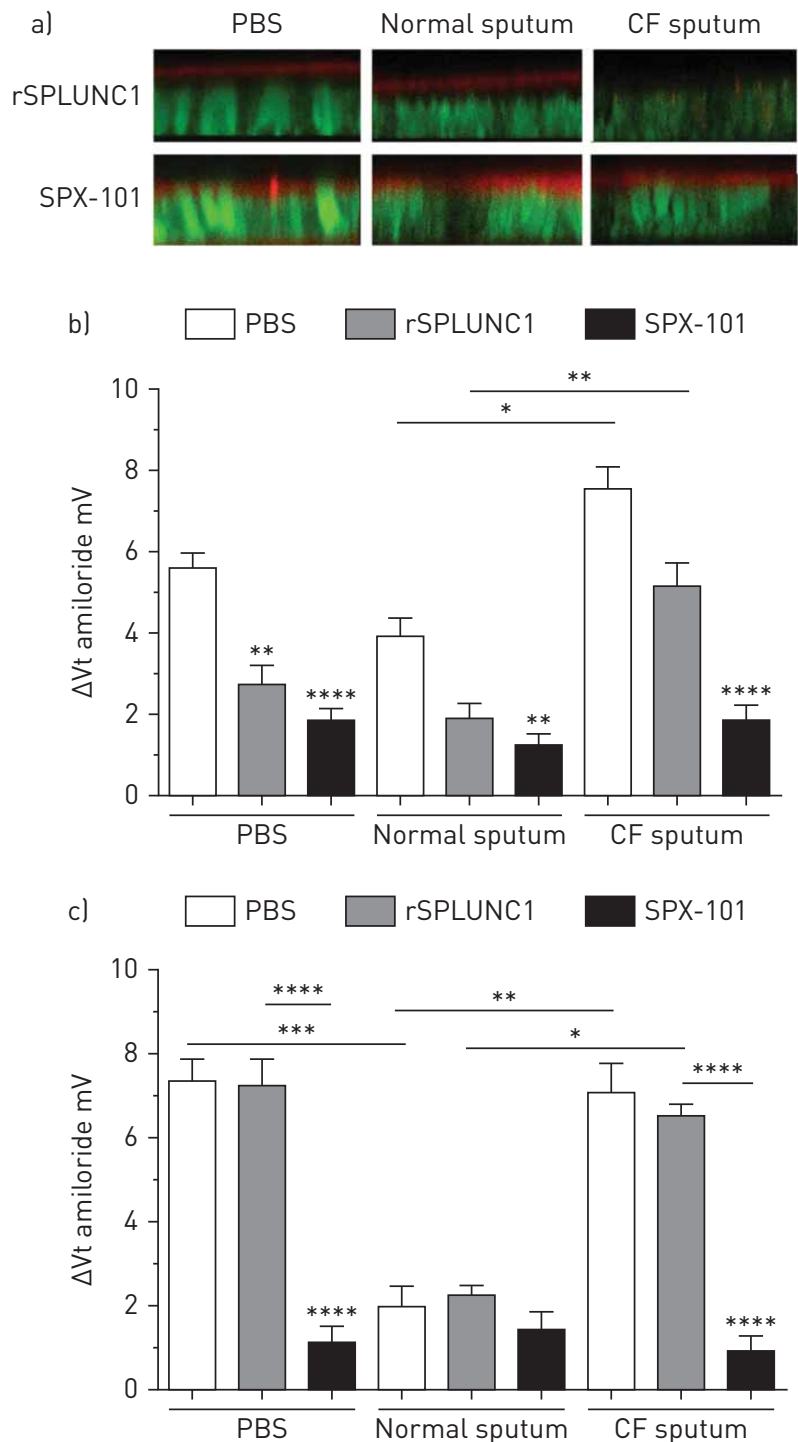

FIGURE 7 Cystic fibrosis (CF) sputum prevents SPLUNC1 binding to human bronchial epithelial cell (HBEC) mucosal surfaces and elevates the amiloride-sensitive transepithelial potential difference $\left(\mathrm{Vt}_{\mathrm{t}}\right)$. a) Dylight633-rSPLUNC1 and TAMRA-SPX-101 were incubated with PBS, normal sputum and CF sputum for $2 \mathrm{~h}$ at $37^{\circ} \mathrm{C}$ and added mucosally to HBECs for an additional $2 \mathrm{~h}$ before imaging. $\mathrm{XZ}$ confocal micrographs show apical binding of Dylight633-rSPLUNC1 and TAMRA-SPX-101 (red) to normal HBECs. HBECs were stained with calcein-acetoxymethyl (green). b, c) $30 \mu \mathrm{M}$ rSPLUNC1 and SPX-101 were incubated with PBS, normal or CF sputum (pooled from $\mathrm{n}=6$ donors) for $12 \mathrm{~h}$ at $37^{\circ} \mathrm{C}$ and added mucosally to HBECs for an additional $2-3 \mathrm{~h}$. Voltage-sensing microelectrodes were then positioned in the airway surface liquid (ASL) by micromanipulator and the amiloride-sensitive $\mathrm{Vt}_{t}$ was measured. All $\mathrm{Vt}_{\mathrm{t}}$ measurements were performed in the presence of basolateral $10 \mu \mathrm{M}$ bumetanide to inhibit $\mathrm{Cl}^{-}$secretion. $\Delta \mathrm{V}_{\mathrm{t}}$ amiloride was then measured in normal (b) and CF (c) HBECs as indicated. Data are presented as mean \pm SEM from $n=16-24$ HBECs cultured from $n=5-8$ individual donors. Data were analysed by ANOVA with Tukey's post hoc analyses; *: $p<0.05$; **: $p<0.01$; ${ }^{* * *}: p<0.001 ;{ }^{* * * *}: p<0.0001$.

\section{Discussion}

The lung mucosal environment contains $\sim 1000$ proteins, of which $\sim 5 \%$ are proteases and their inhibitors [27]. Under normal conditions, anti-proteases prevent excessive NE activity in the airways [28]. In general, SPLUNC1 was relatively stable in normal sputum (figure 1), although in some cases we did observe some degradation (e.g. supplementary figure S2). However, the degradation product was close in size to full-length SPLUNC1 $(>20 \mathrm{kDa})$ and was similar in size to a SPLUNC1-degradation product that we had previously observed after exposing SPLUNC1 to trypsin [29]. Thus, we speculate that serine proteases present in normal ASL or sputum may post-translationally affect SPLUNC1. However, chronic 
neutrophilia in the CF lung lumen significantly increases free NE levels $[8,28,30]$. Similarly, we found significantly higher NE protein levels and activity in CF sputum compared with sputum from normal healthy donors (figure 1). We also observed that SPLUNC1 protein levels were significantly reduced in $\mathrm{CF}$ sputum and that rSPLUNC1 was rapidly degraded in CF sputum (figures 1 and 2). To confirm that SPLUNC1 was degraded, we performed proteomic analysis. NE-specific SPLUNC1 coverage was markedly increased in CF sputum, indicating inflammation-induced degradation. Crucially, intact S18-like peptides that can inhibit $\mathrm{ENaC}$ were absent in $\mathrm{CF}$ sputum. Despite being cleaved by NE, SPLUNC1 remains aggregated for several hours before dissociating [31]. However, the lack of SPLUNC1 coverage in CF sputum and the relatively short half-life of rSPLUNC1 in CF sputum suggest that at steady state SPLUNC1 does not exist as a functional entity in CF airway secretions.

NE inhibitors have previously been tested in CF patients, with little effect. For example, AZD9668 was tested in CF patients and had no effect on inflammation or lung function [32]. Similarly, recombinant $\alpha 1$ anti-trypsin also failed to affect lung inflammation [6]. Our data indicate that sivelestat was only partially effective at preventing SPLUNC1 degradation in CF sputum and this effect was lost over time. ENaC is highly promiscuous and is cleaved by multiple proteases. Thus, there are two possible contributors to the failure of NE inhibitors in the clinic: 1) SPLUNC1 and other proteins are still degraded by CF secretions in the absence of $\mathrm{NE}$; and 2) $\mathrm{ENaC}$ can still be activated by other proteases in the $\mathrm{CF}$ lung leading to persistent ASL dehydration. Thus, whilst newer broad-spectrum anti-proteases such as QUB-TL1 may be more effective at preventing $\mathrm{ENaC}$ cleavage [33], whether or not they are fully effective in the CF lung remains to be determined.

Our working hypothesis is that SPLUNC1 causes removal of ENaC from the plasma membrane, preventing its cleavage and activation. We have recently developed SPX-101, a size-optimised peptide that can inhibit ENaC in CF HBECs and CF-like animal models [18]. Because SPX-101 is currently undergoing clinical trials for the treatment of CF, we tested whether SPX-101 was stable and efficacious in CF sputum samples. Both SPLUNC1 and SPX-101 increased ASL height after overnight incubation in normal sputum. However, after exposure to CF sputum, SPLUNC1 was degraded and failed to regulate ASL height, whereas SPX-101 remained intact and active. As a control, we also incubated SPLUNC1 and SPX-101 overnight with NE and probed for $\alpha$-ENaC-GFP diminution, as an indication of internalisation. Whilst SPLUNC1 lost the ability to inhibit ENaC, SPX-101 again remained active even after prolonged exposure to NE (supplementary material).

SPLUNC1 was degraded in CF sputum from the central airways. However, by utilising SMM, we also observed SPLUNC1 degradation in more distal regions of CF lungs. Consistent with the lack of SPLUNC1, we observed a significant increase in full-length $\alpha$-, $\beta$ - and $\gamma$-ENaC subunits, suggesting that SPLUNC1 had failed to internalise ENaC. Crucially, $\alpha$ - and/or $\gamma$-ENaC must be proteolytically cleaved to be active $[26,34,35]$ and we also detected significant increases in cleaved $\alpha$ - and $\gamma$-ENaC subunits in CF airways. The basolateral $\mathrm{Na}^{+} / \mathrm{K}^{+}$-ATPase serves to pump out $\mathrm{Na}^{+}$that enters the cell via $\mathrm{ENaC}$ in order to keep intracellular $\mathrm{Na}^{+}$at $\sim 23 \mathrm{mM}$ [36]. This pump has previously been shown to be functionally upregulated in CF airways [37]. Similarly, we observed a significant increase in $\mathrm{Na}^{+} / \mathrm{K}^{+}$-ATPase protein levels in CF airways, consistent with the increase in cleaved $\alpha$ - and $\gamma$-ENaC subunits. Welsh and colleagues $[38,39]$ have proposed that the increase in $\mathrm{ENaC}$ activity detected by electrophysiology is an artefact caused by amiloride-induced apical membrane hyperpolarisation. However, our biochemical data, which demonstrate that cleaved (i.e. active) $\mathrm{ENaC}$ as well as the necessary $\mathrm{Na}^{+} / \mathrm{K}^{+}$-ATPase are upregulated in $\mathrm{CF}$ epithelia, suggest that this is not the case.

Whilst defective ion transport is widely accepted to be the initial event in the pathogenesis of CF lung disease, the role of $\mathrm{ENaC}$ remains highly controversial [17]. A number of studies have demonstrated increased electrical $\mathrm{Na}^{+}$flux and fluid changes in $\mathrm{CF}$ airway epithelia that were attributable to increased $\mathrm{ENaC}$ activity [3, 40]. In contrast, others failed to find differences in $\mathrm{CF}$ piglets and in cultured airway epithelia [38, 39, 41]. Our data revealed that rSPLUNC1 failed to bind to HBEC apical membranes after pre-incubation with CF sputum, whereas N-terminally labelled SPX-101 bound equally well after exposure to normal or CF sputum. rSPLUNC1 was amine-labelled with DyLight633, suggesting that multiple residues were labelled. Thus, whilst it is possible that CF proteases may have removed some dye-labelled residues, we limited our SPLUNC1/CF sputum pre-incubation to $2 \mathrm{~h}$, at which point $\sim 20 \%$ of the protein should have remained.

In our microelectrode studies, we first added bumetanide in order to avoid any confounding effects of amiloride-induced $\mathrm{Cl}^{-}$secretion in non-CF HBECs [23]. SPLUNC1 lowered the amiloride-sensitive $\mathrm{Vt}_{\mathrm{t}}$ in non-CF but not CF HBECs, whereas SPX-101 lowed Vt regardless of genotype. Here, the reduced bicarbonate secretion in CF HBECs contributes to the moderate acidification needed to prevent SPLUNC1 function. The requirement of functional CFTR for SPLUNC1 to inhibit ENaC is likely due to the presence 
of $\mathrm{pH}$-sensitive salt bridges in the tertiary structure of SPLUNC1, which render it inactive below $\mathrm{pH} 7$. Conversely, isolated ENaC-inhibitory peptides, such as SPX-101, lack the salt bridges and are $\mathrm{pH}$ independent [11]. Surprisingly, both non-CF and CF HBECs showed a significant decrease in the amiloride-sensitive $\mathrm{Vt}$ after being exposed to normal sputum for $\sim 2 \mathrm{~h}$. This is likely due to the presence of endogenous S18-like ENaC-inhibitory peptides [12]. Indeed, after passing normal sputum through a size-exclusion column to remove proteins $\geqslant 10 \mathrm{kDa}$, including full-length SPLUNC1 which is $\sim 25 \mathrm{kDa}$, the filtrate was able to significantly increase ASL height. Adding rSPLUNC1 to the filtrate had no further effect (supplementary figure S5), suggesting that the filtrate and SPLUNC1 have a similar target (i.e. $\mathrm{ENaC}$ ). Thus, whilst we cannot state that SPLUNC1/S18 is the only ENaC-regulatory peptide in the ASL, our data indicate that small, ASL-regulatory peptides are present in normal sputum. In contrast, adding CF sputum significantly elevated the amiloride-sensitive $\mathrm{Vt}$ in both non-CF and CF HBECs relative to normal sputum. As with the ASL height and binding experiments, SPLUNC1 was ineffective at ameliorating these effects, due to excessive degradation. However, SPX-101 remained active and could significantly reduce the amiloride-sensitive $\mathrm{Vt}$. These changes are likely due to an abundance of proteases, many of which can cleave and activate $\mathrm{ENaC}$, as well as a lack of functional SPLUNC1 or SPLUNC1-derived peptides in CF sputum. Taken together, these findings demonstrate that the CF mucosal environment (i.e. the ASL secretions) influences ion transport in established CF lung disease. Previous studies have demonstrated cellular interactions between CFTR and ENaC $[4,14,15]$. However, our study is the first to test the effects of CF airways secretions on $\mathrm{ENaC}$ activity and our data strongly indicate that the extracellular environment drives $\mathrm{ENaC}$ hyperactivity in $\mathrm{CF}$ airways.

In conclusion, we have shown that SPLUNC1 is absent or markedly reduced in CF airway secretions, which contributes to increased ENaC activity and ASL dehydration in CF HBECs. These data suggest that studying CF HBECs in the presence of CF sputum will serve as a more realistic model of CF airways and also indicate that novel therapies should be tested for efficacy in the presence of CF secretions.

Acknowledgements: We thank the University of Carolina (UNC) Cystic Fibrosis Center Tissue Cores for providing cells and tissue, Colin Bingle (University of Sheffield, Sheffield) for providing the SPLUNC1 construct, Debbie Baines (St. George's, University of London) for providing the $\alpha$-ENaC-GFP construct, Thomas Kleyman (University of Pittsburgh) and M. Jackson Stutts (UNC) for the $\beta$ - and $\gamma$-ENaC constructs, and Michael Miley and Ricard Feng (UNC) for purifying SPLUNC1. Finally, we would like to thank Brian Button (UNC) for allowing use of his equipment for this project.

Author contributions: M.J. Webster, B. Reidel, C.D. Tan, A. Ghosh and R. Tarran designed and performed research and analysed data. N.E. Alexis, S.H. Donaldson and C.M.P. Ribeiro provided sputum and SMM samples, designed experiments and interpreted data. M. Kesimer designed experiments and analysed data. M. J. Webster, B. Reidel and R. Tarran wrote the manuscript. All other authors edited and approved the manuscript.

Support statement: This study was funded by National Institutes of Health R01HL108927, P30DK065988, the UK Cystic Fibrosis Trust (INOVCF), Cystic Fibrosis Foundation R026-CR11 and TARRAN17G0. Funding information for this article has been deposited with the Crossref Funder Registry.

Conflict of interest: M.J. Webster has nothing to disclose. B. Reidel has nothing to disclose. C.D. Tan has nothing to disclose. A. Ghosh has nothing to disclose. N.E. Alexis has nothing to disclose. S.H. Donaldson reports grants from Vertex Pharmaceuticals and Parion Sciences, grants and personal fees from Nivalis Pharmaceuticals, and personal fees from Pulmatrix, Novartis Pharmaceuticals and PTC Therapeutics, outside the submitted work; in addition, S.H. Donaldson has a patent "Regulation of sodium channels by PLUNC proteins" with royalties paid. M. Kesimer has nothing to disclose. C.M.P. Ribeiro is founder of and has equity in Irex Pharma. R. Tarran is founder of and on the board of directors of Spyryx Biosciences, outside the submitted work; in addition, R. Tarran has a patent "Regulation of sodium channels by PLUNC proteins" with royalties paid.

\section{References}

Quinton PM. Chloride impermeability in cystic fibrosis. Nature 1983; 301: 421-422.

2 Matalon S, Bartoszewski R, Collawn JF. Role of epithelial sodium channels in the regulation of lung fluid homeostasis. Am J Physiol Lung Cell Mol Physiol 2015; 309: L1229-L1238.

3 Boucher RC, Stutts MJ, Knowles MR, et al. Na+ transport in cystic fibrosis respiratory epithelia. Abnormal basal rate and response to adenylate cyclase activation. J Clin Invest 1986; 78: 1245-1252.

4 Stutts MJ, Canessa CM, Olsen JC, et al. CFTR as a cAMP-dependent regulator of sodium channels. Science 1995; 269: 847-850.

5 Matsui $\mathrm{H}$, Grubb BR, Tarran R, et al. Evidence for periciliary liquid layer depletion, not abnormal ion composition, in the pathogenesis of cystic fibrosis airways disease. Cell 1998; 95: 1005-1015.

6 Martin SL, Moffitt KL, McDowell A, et al. Association of airway cathepsin B and S with inflammation in cystic fibrosis. Pediatr Pulmonol 2010; 45: 860-868.

7 Chalmers JD, Moffitt KL, Suarez-Cuartin G, et al. Neutrophil elastase activity is associated with exacerbations and lung function decline in bronchiectasis. Am J Respir Crit Care Med 2017; 195: 1384-1393.

8 Dittrich AS, Kühbandner I, Gehrig S, et al. Elastase activity on sputum neutrophils correlates with severity of lung disease in cystic fibrosis. Eur Respir J 2018; 51: 1701910. 
9 Wu T, Huang J, Moore PJ, et al. Identification of BPIFA1/SPLUNC1 as an epithelium-derived smooth muscle relaxing factor. Nat Commun 2017; 8: 14118.

10 Bartlett J, Gakhar L, Penterman J, et al. PLUNC: a multifunctional surfactant of the airways. Biochem Soc Trans 2011; 39: 1012-1026.

11 Garland AL, Walton WG, Coakley RD, et al. Molecular basis for $\mathrm{pH}$-dependent mucosal dehydration in cystic fibrosis airways. Proc Natl Acad Sci USA 2013; 110: 15973-15978.

12 Hobbs CA, Blanchard MG, Alijevic O, et al. Identification of the SPLUNC1 ENaC-inhibitory domain yields novel strategies to treat sodium hyperabsorption in cystic fibrosis airway epithelial cultures. Am J Physiol Lung Cell Mol Physiol 2013; 305: L990-L1001.

13 Kim CS, Ahmad S, Wu T, et al. SPLUNC1 is an allosteric modulator of the epithelial sodium channel. FASEB $J$ 2018; 32: 2478-2491

14 Schreiber R, Hopf A, Mall M, et al. The first-nucleotide binding domain of the cystic-fibrosis transmembrane conductance regulator is important for inhibition of the epithelial Na+ channel. Proc Natl Acad Sci USA 1999; 96: 5310-5315.

15 Rubenstein RC, Lockwood SR, Lide E, et al. Regulation of endogenous ENaC functional expression by CFTR and AF508-CFTR in airway epithelial cells. Am J Physiol Lung Cell Mol Physiol 2011; 300: L88-L101.

16 Stoltz DA, Meyerholz DK, Pezzulo AA, et al. Cystic fibrosis pigs develop lung disease and exhibit defective bacterial eradication at birth. Sci Transl Med 2010; 2: 29ra31.

17 Stoltz DA, Meyerholz DK, Welsh MJ. Origins of cystic fibrosis lung disease. N Engl J Med 2015; 372: 1574-1575.

18 Scott DW, Walker MP, Sesma J, et al. SPX-101 is a novel epithelial sodium channel-targeted therapeutic for cystic fibrosis that restores mucus transport. Am J Respir Crit Care Med 2017; 196: 734-744.

19 Abdullah LH, Coakley R, Webster MJ, et al. Mucin production and hydration responses to mucopurulent materials in normal versus CF airway epithelia. Am J Respir Crit Care Med 2017; 197: 481-491.

20 Fulcher ML, Randell SH. Human nasal and tracheo-bronchial respiratory epithelial cell culture. Methods Mol Biol 2013; 945: 109-121.

21 Wisniewski JR, Zougman A, Nagaraj N, et al. Universal sample preparation method for proteome analysis. Nat Methods 2009; 6: 359-362.

22 Kesimer M, Cullen J, Cao R, et al. Excess secretion of gel-forming mucins and associated innate defense proteins with defective mucin un-packaging underpin gallbladder mucocele formation in dogs. PLoS One 2015; 10: e0138988.

23 Tarran R, Trout L, Donaldson SH, et al. Soluble mediators, not cilia, determine airway surface liquid volume in normal and cystic fibrosis superficial airway epithelia. J Gen Physiol 2006; 127: 591-604.

24 Jiang D, Wenzel SE, Wu Q, et al. Human neutrophil elastase degrades SPLUNC1 and impairs airway epithelial defense against bacteria. PLoS One 2013; 8: e64689.

25 Sagel SD, Sontag MK, Wagener JS, et al. Induced sputum inflammatory measures correlate with lung function in children with cystic fibrosis. J Pediatr 2002; 141: 811-817.

26 Caldwell RA, Boucher RC, Stutts MJ. Neutrophil elastase activates near-silent epithelial $\mathrm{Na}^{+}$channels and increases airway epithelial $\mathrm{Na}^{+}$transport. Am J Physiol Lung Cell Mol Physiol 2005; 288: L813-L8L9.

27 Kesimer M, Kirkham S, Pickles RJ, et al. Tracheobronchial air-liquid interface cell culture: a model for innate mucosal defense of the upper airways? Am J Physiol Lung Cell Mol Physiol 2009; 296: L92-L100.

28 Birrer P, McElvaney NG, Rüdeberg A, et al. Protease-antiprotease imbalance in the lungs of children with cystic fibrosis. Am J Respir Crit Care Med 1994; 150: 207-213.

29 Garcia-Caballero A, Rasmussen JE, Gaillard E, et al. SPLUNC1 regulates airway surface liquid volume by protecting ENaC from proteolytic cleavage. Proc Natl Acad Sci 2009; 106: 11412-11417.

30 Armstrong DS, Grimwood K, Carlin JB, et al. Lower airway inflammation in infants and young children with cystic fibrosis. Am J Respir Crit Care Med 1997; 156: 1197-1204.

31 Ahmad S, Tyrrell J, Walton WG, et al. Short Palate, Lung, and Nasal Epithelial Clone 1 has antimicrobial and antibiofilm activities against the Burkholderia cepacia complex. Antimicrob Agents Chemother 2016; 60: 6003-6012.

32 Elborn JS, Perrett J, Forsman-Semb K, et al. Efficacy, safety and effect on biomarkers of AZD9668 in cystic fibrosis. Eur Respir J 2012; 40: 969-976.

33 Reihill JA, Walker B, Hamilton RA, et al. Inhibition of protease-epithelial sodium channel signaling improves mucociliary function in cystic fibrosis airways. Am J Respir Crit Care Med 2016; 194: 701-710.

34 Vallet V, Chraibi A, Gaeggeler HP, et al. An epithelial serine protease activates the amiloride-sensitive sodium channel. Nature 1997; 389: 607-610.

35 Kleyman TR, Kashlan OB, Hughey RP. Epithelial $\mathrm{Na}^{+}$channel regulation by extracellular and intracellular factors. Annu Rev Physiol 2018; 80: 263-281.

36 Boucher RC. Human airway ion transport. Part one. Am J Respir Crit Care Med 1994; 150: 271-281.

37 Stutts MJ, Knowles MR, Gatzy JT, et al. Oxygen consumption and ouabain binding sites in cystic fibrosis nasal epithelium. Pediatr Res 1986; 20: 1316-1320.

38 Itani $\mathrm{OA}$, Chen $\mathrm{JH}$, Karp $\mathrm{PH}$, et al. Human cystic fibrosis airway epithelia have reduced Cl- conductance but not increased $\mathrm{Na}^{+}$conductance. Proc Natl Acad Sci USA 2011; 108: 10260-10265.

39 Chen JH, Stoltz DA, Karp PH, et al. Loss of anion transport without increased sodium absorption characterizes newborn porcine cystic fibrosis airway epithelia. Cell 2010; 143: 911-923.

40 Knowles M, Gatzy J, Boucher R. Increased bioelectric potential difference across respiratory epithelia in cystic fibrosis. N Engl J Med 1981; 305: 1489-1495.

41 Davies JC, Davies M, McShane D, et al. Potential difference measurements in the lower airway of children with and without cystic fibrosis. Am J Respir Crit Care Med 2005; 171: 1015-1019. 